\title{
Finite element simulation of bone remodelling in human mandible around osseointegrated dental implant
}

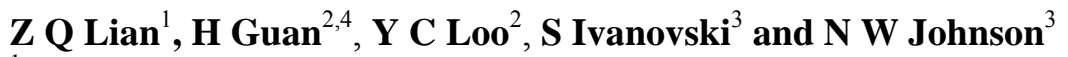 \\ ${ }^{1}$ State Key Laboratory of Structural Analysis for Industrial Equipment, Department of \\ Engineering Mechanics, Dalian University of Technology, Dalian, 116023, China \\ ${ }^{2}$ Griffith School of Engineering, Griffith University Gold Coast Campus, Queensland \\ 4222, Australia \\ ${ }^{3}$ School of Dentistry and Oral Health, Griffith University Gold Coast Campus, \\ Queensland 4222, Australia \\ E-mail: $\underline{\text { h.guan@griffith.edu.au }}$
}

\begin{abstract}
Modern dental implant is a biocompatible titanium device surgically placed into a jawbone to support a prosthetic tooth crown in order to replace missing teeth. Implants are superior to conventional prostheses, in both function and long-term predictability. However, placement of an implant changes the normal mechanical environment of jawbone, which causes the bone density to redistribute and adapt to the new environment through a process of remodelling. This study aims to predict the density distribution in human jawbone around osseointegrated dental implant. Based on two popular, yet distinctive theories for bone remodelling, a new remodelling algorithm is proposed. The proposed algorithm is verified by a two-dimensional (2D) plate model. Then, a 2D finite element model of implant and jawbone is studied. The effects of two parameters, viz the reference value of strain energy density (SED) and 'lazy zone' region, on density distribution, are also examined. This study has demonstrated that consideration of the lazy zone, is less important than consideration of the stress and strain (quantified as SED) induced within the bone. Taking into account both 'lazy zone' effect and self-organisational control process, the proposed bone remodelling algorithm has overcome the shortcomings of the two existing theories.
\end{abstract}

\section{Introduction}

Development of an ideal substitute for missing teeth has been a major aim of dentistry for millennia. For 100 years, missing teeth have been replaced by "conventional" prostheses (fixed or removable), fabricated from plastic and/or metal alloys and supported by the remaining teeth and/or the soft tissues. These are often unsatisfactory because of lack of retention and/or psychological inability to accept removable appliances - or because of damage to remaining teeth and/or soft tissues. In these cases, prostheses retained by an implant are attractive. Implant bone restorations have become a clinically and scientifically accepted treatment modality over the past 30 years. The realization that implants made of commercially pure titanium attain direct bone to implant contact initiated this revolution in oral rehabilitation. This phenomenon of osseointegration was first described by Branemark et al. [1] and Schroeder et al. [2].

\footnotetext{
${ }^{4}$ To whom any correspondence should be addressed.
} 
As a living tissue, bone undergoes continuous adaptation and self-repair by resorption and formation, and is capable of optimising its internal structure under the influence of external loads. This self-adaptation process, or 'remodelling', has an enormous effect on the overall behavior of the entire bone tissue throughout an individual's lifetime. Following an early publication of Wolff [3], many theories describing the bone-adaptation process have been proposed. Carter [4] suggested that bone is 'lazy' in terms of poor reaction to mechanical signals, and there was recognition of the existence of a 'lazy zone' representing the range of stimulus within which no remodelling occurs. The concept of 'lazy zone', recognising that a threshold stimulus needed to be exceeded for bone adaptation to occur, was incorporated by Huiskes et al. [5] who used the term strain energy density (SED) to describe the stresses and strains that control bone remodelling through bone formation and resorption. This theory has been developed further by Weinans et al. [6] and Turner et al. [7]. Mullender et al. [8] and Mullender and Huiskes [9] suggested a physiological approach to simulate bone remodelling assuming that osteocytes are sensitive to mechanical loading and are active in controlling bone adaptation.

Computer simulation of bone remodelling generally involves finite element analysis for calculating stresses, strains and SEDs within the bone thus determining how bone adapts itself in response to mechanical stimuli. The finite element method (FEM) was initially introduced to orthopedic biomechanics by Brekelmans et al. [10] and Rybicki et al. [11] to evaluate the stresses in human bone. Since then, the method has been successfully applied for stress analyses of bone and bone-related tissue. The above-mentioned theories of bone remodelling have been used successfully in conjunction with the FEM to predict density distribution in proximal femur $[8,9,12]$ and bone adaptation following hip prosthetic implantation [5,13-16]. While these theories were essentially developed for orthopedics, they are generally applicable to jawbone surrounding a dental implant [17]. However, work in this area is limited. Mellal et al. [18] used three different stimuli (equivalent strain, equivalent stress and SED) to predict bone remodelling around a dental implant based on the existing theories and the results were compared with in vivo data. Li et al. [19] developed a new bone remodelling algorithm by introducing an additional quadratic term based on the theory of Weinans et al. [6], which can simulate both underload and overload resorption. The algorithm was applied in conjunction with the FEM to a practical case of dental implant treatment. Under steady conditions, no overall changes in bone would be observable due to balance in resorption and formation. However when the implant is placed into mandible, the mechanical environment of jawbone changes accordingly, hence the balance of bone resorption and formation is no longer maintained. From a clinical viewpoint, better understanding of the way that functional forces can affect the remodelling of bone is important in terms of identifying minimal and maximal loading forces required for the maintenance of stable osseointegration during function, as well as preventing overload that can lead to implant failure.

This paper aims to propose a new algorithm for bone remodelling based on the existing theories of Huiskes et al. [5], Weinans et al. [6], Mullender et al. [8] and Mullender and Huiskes [9]. The proposed algorithm is verified by a 2D plate model studied previously by Weinans et al. [6] and Mullender et al. [8], which confirms its accuracy and reliability. To demonstrate the ability of the proposed algorithm in predicting the density distribution of bone surrounding a dental implant, a 2D FE model of implant and jawbone is studied. The effects of two parameters viz the reference value of SED and 'lazy zone' region, on density distribution are also examined and discussed in some detail.

\section{The existing bone remodelling theories}

Among a number of bone remodelling theories [5-9,12,13,20-23], two popular yet distinctive ones are summarised herein.

\subsection{Huiskes and Weinans Theory}

A popular bone remodelling theory suggested by Huiskes et al. [5] and Weinans et al. [6] states that: 


$$
\frac{d \rho}{d t}=\left\{\begin{array}{ccc}
B\left(\frac{U}{\rho}-(1+s) K\right) & \text { if } & \frac{U}{\rho}>(1+s) K \\
0 & \text { if } & (1-s) K \leq \frac{U}{\rho} \leq(1+s) K \\
B\left(\frac{U}{\rho}-(1-s) K\right) & \text { if } & \frac{U}{\rho}<(1-s) K
\end{array}\right.
$$

where $d \rho / d t$ is the rate of change in density; $\rho$ is the apparent density of bone tissue; $B$ is a constant regulating the rate of the remodelling process; $s$ is in percentage denoting the region of the 'lazy zone' around the threshold value $K$, which is a reference value for SED (see figure 1); the 'lazy zone' describes the bone not having a net change in apparent density, and is defined as $U / \rho ; U$ is the SED which can be expressed as:

$$
U=\frac{1}{2} \sigma \cdot \varepsilon
$$

where $\sigma$ and $\varepsilon$ are respectively the stress and strain tensors of the bone tissue.

For multiple-load cases, the SED can be expressed as:

$$
U=\frac{1}{n} \sum_{i=1}^{n} U_{i}
$$

where $n$ is the number of load cases and $U_{i}$ is the SED under load case $i$.

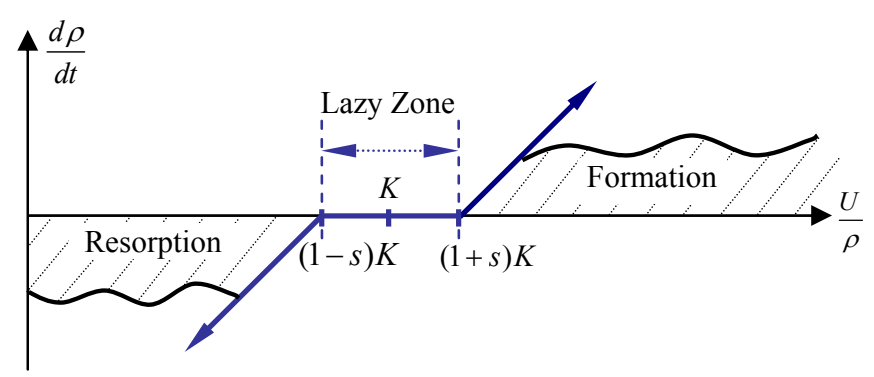

Figure 1. The assumed local bone adaptation as a function of SED with 'lazy zone' effect

This algorithm was applied to predict the density distribution in normal proximal femur with results showing a discontinuous pattern [6]. As discussed by Ruimerman and Huiskes [24], only when the density distributions were locally averaged from discontinuous density patterns in the femoral head where the trabecular bone is located, the resulting density distributions correlate well with those in a real proximal femur. This algorithm showed an unstable condition in its mathematical operation. Due to the nature of differential equations used in the algorithm to describe the adaptive remodelling process, the simulation produces discontinuous configurations, a phenomenon called 'checker-board'.

\subsection{Mullender et al. and Mullender and Huiskes Theory}

Not having intrinsically different biological nature from the theory of Huiskes et al. [5] and Weinans et al. [6], Mullender et al. [8] and Mullender and Huiskes [9] suggested a new algorithm which simulated bone remodelling as a self-organisational control process. In their theory, the bone is assumed to have $N$ sensor cells distributed uniformly over its volume. An arbitrary sensor $i$ measures a signal $\mathrm{Si}$, which is given as:

$$
S_{i}=\frac{U_{i}}{\rho_{i}}
$$


where $U_{i}$ is the SED and $\rho_{i}$ is the density at the location of the sensor. The density $\rho(\mathbf{x}, t)$ at location $\mathbf{x}$ is regulated by the stimulus value $\Phi(\mathbf{x}, t)$, to which all sensor cells contribute, relative to their distance from $\mathbf{x}$. Hence,

$$
\Phi(\mathbf{x}, t)=\sum_{i=1}^{N} f_{i}(\mathbf{x})\left(S_{i}-K\right)
$$

where $N$ is the total number of sensors; $K$ is a reference signal of SED and $f_{i}(\mathbf{x})$ is a spatial influence function given as:

$$
f_{i}(\mathbf{x})=e^{-\left[d_{i}(\mathbf{x}) / D\right]}
$$

where $d_{i}(\mathbf{x})$ is the distance between sensor $i$ and location $\mathbf{x}, D$ is the rate of the spatial influence reduction. Note that $D$ represents the distance from a sensor at which location its effect is reduced to $e^{-1}$, i.e. $36.8 \%$. This is illustrated in figure 2 .

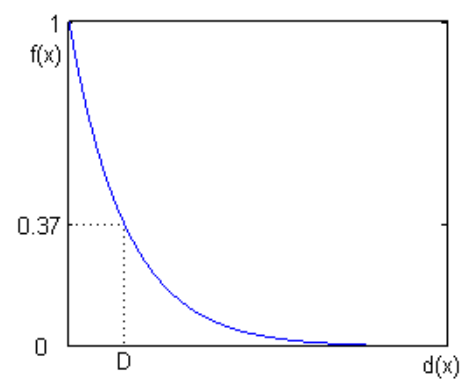

Figure 2. Relationship between $f(\mathrm{x})$ and $d(\mathrm{x})$. Figure 3. 2D plate model simulating bone $\mathrm{t}$ (Carter and Hayes [26], Weinans et al. [6]).

The density $\rho(x, t)$ is now governed by the rate:

$$
\frac{d \rho(\mathbf{x}, t)}{d t}=\tau \Phi(\mathbf{x}, t), \text { with } 0<\rho(x) \leq \rho_{\max }
$$

where $\rho_{\max }$ is the maximum density of cortical bone, and $\tau$ is a time constant regulating the rate of the process.

This algorithm was used to predict the density distribution for a 2D plate model previously studied by Mullender et al. [8]. The model produced trabecular-like structures without the 'checker-board' patterns. It should be noted that the checker-board effect produced by the phenomenological models like the one of Weinans et al. [6] has been completely solved by different techniques including extrapolation to the nodes [23] or using non-local stimuli [8,9]. The checker-board phenomenon is believed to be attributable to the softening induced by resorption rather than remodelling itself. When using the non-local definition of the stimulus as a sum of the signal of different osteocytes near the point under concerned, Mullender et al.'s model [8,9] does not produce 'checker-board' patterns.

\section{The proposed algorithm}

The above-mentioned two existing theories have both shown to exhibit advantages and disadvantages. Huiskes and Weinans's theory takes into account the 'lazy zone' effect but it is unstable in its mathematical operation leading to a discontinuous and an inevitable 'checker board' pattern. Mullender's theory, on the other hand, is physiologically and mechanically more consistent and the 'checker board' phenomena can be effectively eliminated. However the effect of 'lazy zone' was not considered. The 'lazy zone' effect, initially proposed by Carter [4] based on experimental 
investigation, has been verified by Rubin and Lanyon [25] to be an important factor which should be considered in the simulation of the remodelling process.

To overcome the shortcomings of the above two theories, a new algorithm combing these two theories is proposed herein. For an arbitrary sensor $i$,

$$
\frac{d \rho_{i}(\mathbf{x}, t)}{d t}=\tau \Phi_{i}(\mathbf{x}, t)
$$

and

$$
\Phi_{i}(\mathbf{x}, t)=\left\{\begin{array}{ccc}
\sum_{i=1}^{N} f_{i}(\mathbf{x})\left(\frac{U_{i}}{\rho_{i}}-(1+s) K\right) & \text { if } & \frac{U_{i}}{\rho_{i}}>(1+s) K \\
0 & \text { if } & (1-s) K \leq \frac{U_{i}}{\rho_{i}} \leq(1+s) K, \text { with } 0<\rho_{i}(x) \leq \rho_{\max } \\
\sum_{i=1}^{N} f_{i}(\mathbf{x})\left(\frac{U_{i}}{\rho_{i}}-(1-s) K\right) & \text { if } & \frac{U_{i}}{\rho_{i}}<(1-s) K
\end{array}\right.
$$

where $f_{i}(\mathbf{x})$ is the spatial influence function in the same form as in equation (6).

In finite element simulation, it is assumed that each element contains one sensor which is located at the center of the element. As the apparent density of bone tissue changes during the process of remodelling, the Young's modulus changes accordingly. Some empirical relationships between the apparent density and the mechanical properties of bone have been established experimentally [26-29]. The Young's modulus $E$ at location $\mathbf{x}$ is calculated according to Currey [27] and Rice et al. [29] as

$$
E(\mathbf{x}, t)=C \rho(\mathbf{x}, t)^{\gamma}
$$

where $\rho$ is the apparent density of bone tissue; $C$ and $\gamma$ are the constants.

In the iterative finite element analysis process, the governing equation (8) is necessary to be rewritten as:

$$
\rho_{i}(t+\Delta t)=\rho_{i}(t)+\Delta t \tau \Phi_{i}(t)
$$

where $\Delta t$ is the time step in the iteration process; $\Phi(t)$ is in the same form as in equation (9). The iteration is continued until no more significant changes occur in density distribution.

\section{Verification}

The proposed algorithm is verified against a 2D plate model (see figure 3), studied by Weinans et al. [6] and Mullender et al. [8] to simulate the density distribution within a bone tissue. All the parameters are the same as those adopted by Weinans et al. [6] and Mullender et al. [8]. For the purpose of comparison, the value $s$ is set to zero meaning that the effect of 'lazy zone' is not considered in the analysis.

Figure 4 compares the density distribution outcomes due to Mullender et al.'s [8] theory and the proposed algorithm. The predicted result shown in figure $4 \mathrm{~b}$ bears a strong resemblance to that of Mullender et al. (figure 4a), confirming the accuracy of the proposed algorithm. To evaluate the effect of 'lazy zone', a further analysis is carried out by setting $s=0.1$ while other parameters remain unchanged. When the 'lazy zone' effect is considered (figure $4 \mathrm{c}, s=0.1$ ), the pattern of density distribution becomes smoother in shape as compared to that of $s=0$ (figure $4 \mathrm{~b}$ ), although the morphology is almost the same. The total area of grey regions (representing cancellous bone) is larger in figure $4 \mathrm{c}$ than in figure $4 \mathrm{~b}$ suggesting that less bone adaptation (viz resorption and formation) occurs if the 'lazy zone' effect is considered.

The predicted density distributions for the plate model agree well with the Mullender's results, confirming the reliability of the proposed algorithm when taking into account both the 'lazy zone' 
effect and mathematical stability. The proposed algorithm is hence applied to simulate density distribution within a mandibular bone surrounding a dental implant.

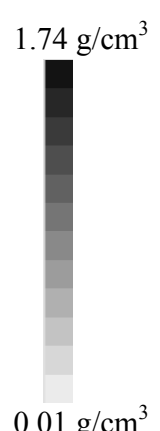

$0.01 \mathrm{~g} / \mathrm{cm}^{3}$

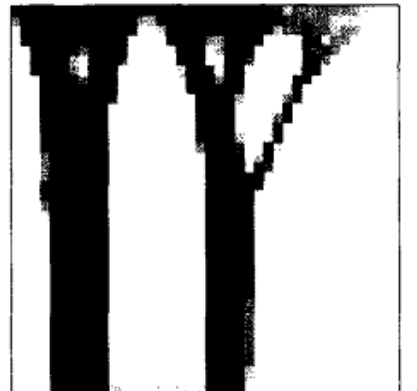

(a)

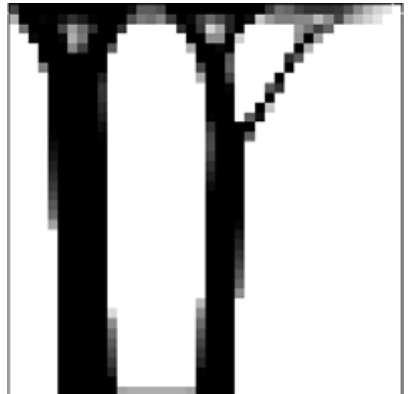

(b)

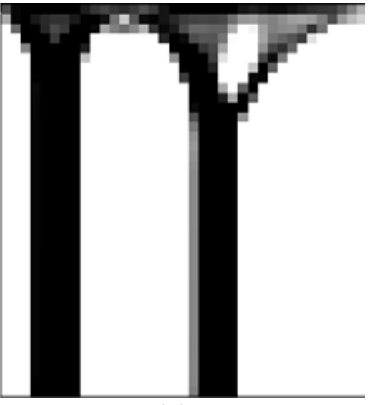

(c)

Figure 4. Comparison of density distribution: (a) Mullender et al.'s [8] prediction with s=0; (b) predicted result with $\mathrm{s}=0$; (c) predicted result with $\mathrm{s}=0.1$.

\section{Simulation of density distribution in mandibular bone}

Studied herein is a 2D representation of an implant and mandible. The modelling scheme follows that of a previous study [30]. It should be noted that this is a preliminary study dealing with a new remodelling algorithm aimed at overcoming the limitations of the existing methodologies. For the purpose of initial verification, 2D analysis is undertaken to obtain fundamental understanding of the remodelling process. 3D analysis will be conducted in the future so that detailed implant geometry can be considered and more accurate solutions be obtained.

Different types of bone, i.e. cortical and cancellous bone are identified in the model based on CT images. The implant dimensions shown in figure 5a are based on those of Neoss implant (Neoss Limited, UK). The implant is conical with 2 degrees of taperage and has a helical thread. The load and boundary conditions shown in figure $5 \mathrm{~b}$ are based on the work of Chou et al. [31] in which the implant system is loaded with an occlusal load of $100 \mathrm{~N}$ on the crown at an angle of $11^{\circ}$ and a uniformly distributed pressure of $500 \mathrm{kPa}$ on the outer surface of the cortical bone. The pressure load is applied to simulate more realistically the boundary conditions.

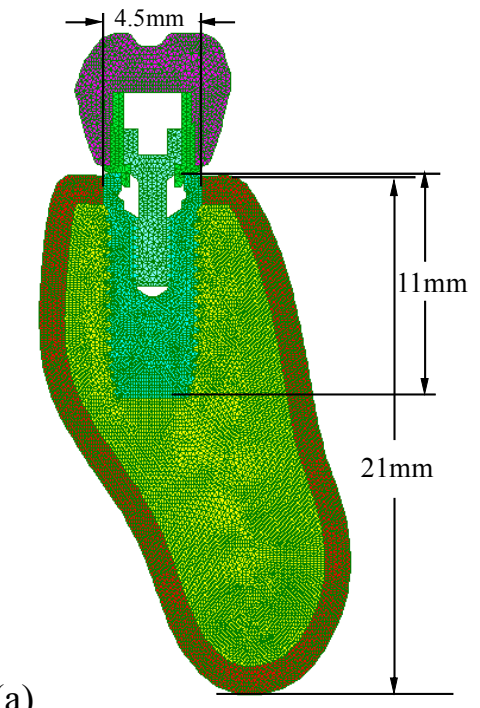

(a)

Figure 5. Finite element model of implant and jawbone: (a) dimensions; (b) load, boundary conditions and material properties.

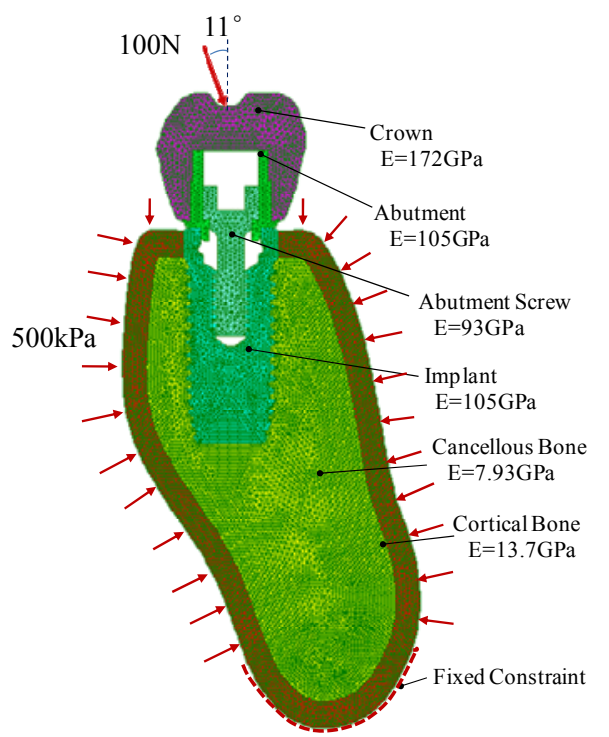

(b) 
An extensive literature review by van Staden et al. [32] indicates that the assumed range of Young's modulus for cortical bone varies from 5.57 to $22.8 \mathrm{GPa}$ and that of cancellous bone from 0.08 to $7.93 \mathrm{GPa}$. Typical values of Young's modulus $(E)$ of bone and implant for the analysis are selected based on the work of Papavasiliou et al. [33], which are also detailed in figure 5b. The Poisson's ratio for all components is taken as 0.3 . The bone tissue and implant are assumed to be isotropic materials. Note that in this study a perfect connection between the implant and jawbone is assumed.

The remodelling is considered for cancellous bone only with a uniform initial density distribution of $\rho=1.4 \mathrm{~g} / \mathrm{cm}^{3}$ which corresponds to $E=7.93 \mathrm{GPa}$. In this case, a specific relationship between $E$ and density $\rho$ is chosen based on Carter and Hayes' study [26], i.e:

$$
E(x, t)=3790 \times \rho^{3}
$$

where $\mathbf{x}$ is the location of sensors; $t$ is the remodelling time. The maximum and minimum densities are respectively $\rho_{\max }=1.5347 \mathrm{~g} / \mathrm{cm}^{3}$ and $\rho_{\min }=0.064 \mathrm{~g} / \mathrm{cm} 3$ which corresponds to Young's modulus of 13.7 $\mathrm{GPa}$ and $1 \mathrm{kPa}$. The 'lazy zone' effect $s$ in equation (9) is taken as 0.1 , the constant $\tau=1\left(\mathrm{~g} / \mathrm{cm}^{3}\right)^{2} /(\mathrm{MPa}$ time-unit). The influence parameter $D=0.25 \mathrm{~mm}$.

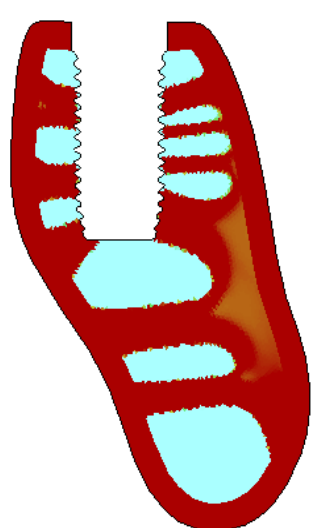

(a)

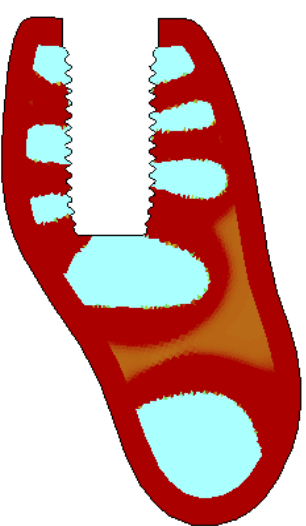

(b)

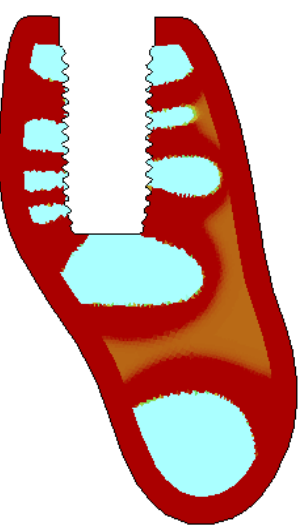

(c)

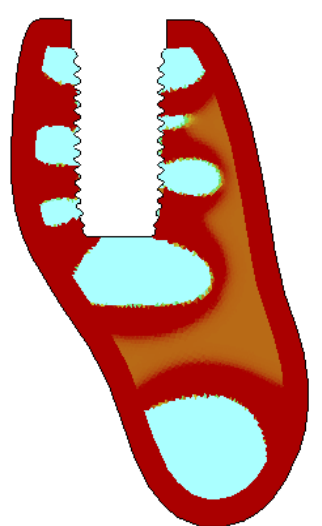

(d)

Figure 6. Influence of 'lazy zone' effect on the density distribution of jawbone surrounding dental implant: (a) $\mathrm{s}=0.1 ;$ (b) $\mathrm{s}=0.2$; (c) $\mathrm{s}=0.3$; (d) $\mathrm{s}=0.4$.

To evaluate the effect of 'lazy zone' on the density distribution, different values of $s$ are considered with a constant SED value $K=0.0052 \mathrm{~J} / \mathrm{g}$. The predicted results are shown in figure 6 . When parameter $s$ increases, the region of 'lazy zone' expands accordingly - hence more bone tissue remains 'lazy', i.e. neither resorption nor formation. This corresponds to a larger 'grey' region on the lingual side of the implant (figure 6d). Although more 'lazy zone' is evident when $s$ increases, the overall morphology does not vary significantly.

Different reference values $K$ in equation (9) are also considered to evaluate the effects of threshold value of SED on density distribution outcomes. The results shown in figure 7 indicate that when $K$ increases, more bone tissue is resorbed in the surrounding jawbone, especially on the lingual side of the implant due to the oblique loading condition.

Figure 8 shows the relationship between the bone mass (for cancellous bone only) and the two parameters $s$ and $K$. When $s$ increases by $300 \%$, the mass only increases by $2.08 \%$. On the other hand, when $K$ increases by $16.7 \%$, the mass decreases by $4.5 \%$. It can be deduced that the bone mass increases slightly with an increase in the 'lazy zone' effect $s$ (figure 8a) and decreases more noticeably with an increase in the reference value $K$ (figure $8 \mathrm{~b}$ ). Hence the parameter $K$ has more significant effect than $s$ on bone remodelling outcomes. 


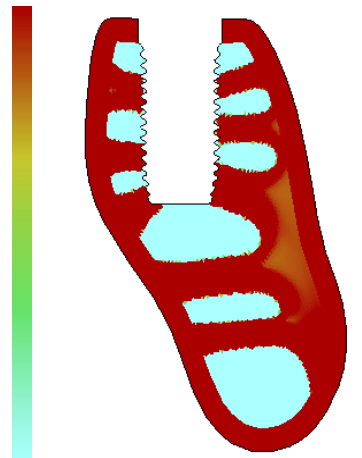

(a)

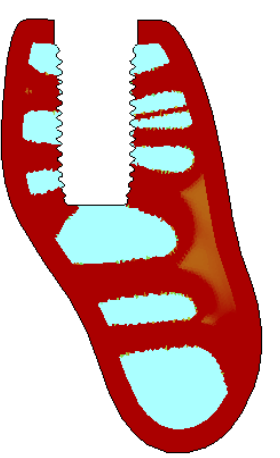

(b)

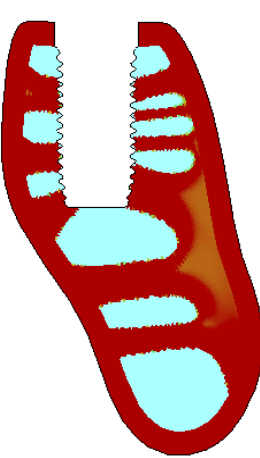

(c)

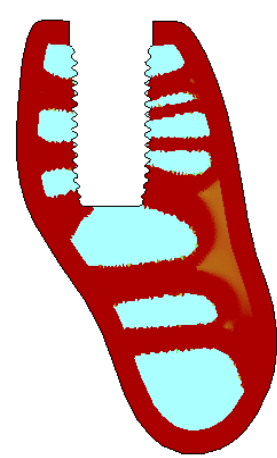

(d)

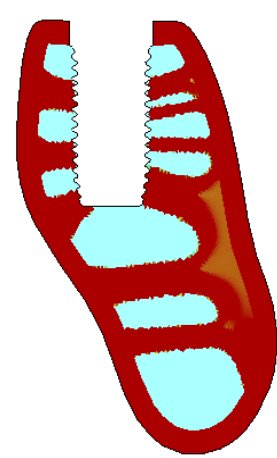

(e)

Figure 7. Influence of reference SED value $K$ on the density distribution of jawbone surrounding dental implant: (a) $K=0.0048 \mathrm{~J} / \mathrm{g}$; (b) $K=0.005 \mathrm{~J} / \mathrm{g}$; (c) $K=0.0052 \mathrm{~J} / \mathrm{g}$; (d) $K=0.0054 \mathrm{~J} / \mathrm{g}$; (e) $K=0.0056 \mathrm{~J} / \mathrm{g}$.

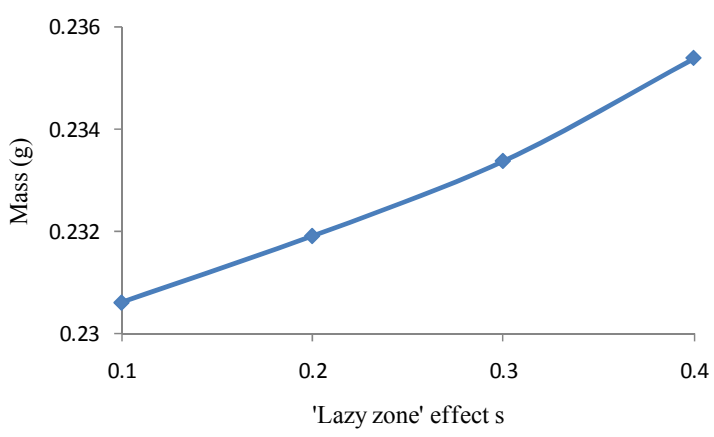

(a)

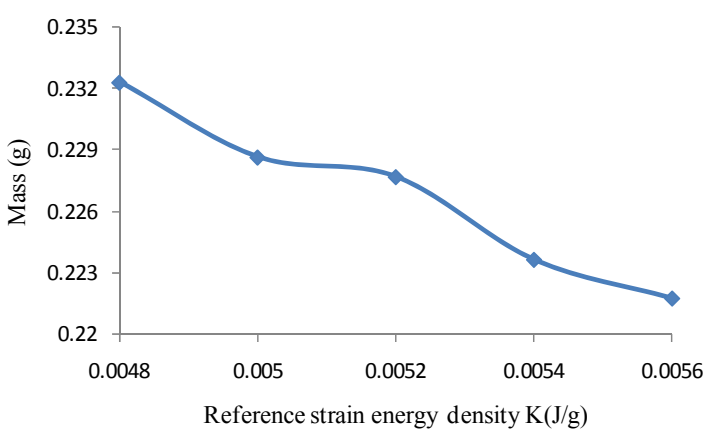

(b)

Figure 8. Influence of two parameters on bone mass: (a) 'lazy zone' effect $s$; (b) reference value $K$.

\section{Discussion}

The proposed algorithm takes into account both the 'lazy zone' effect and the self-organisational control process. It is shown to be accurate and reliable upon verification with a plate model. The algorithm is then used to predict density distribution in human jawbone surrounding a dental implant. The influence of two parameters viz the reference SED value $K$ and the 'lazy zone' effect $s$ on density distribution is also examined. The results indicate that $K$ has more significant influence than $s$.

With an increasing value of $K$, a reduction in bone formation and an increase in bone resorption are evident, and vice versa. There is no general rule or criterion in published literature on selection of the reference value $K$. It is found in this study that a median value among all the SEDs is appropriate to yield a satisfactory density distribution outcome.

Figure 9 compares the predicted density distribution in cancellous bone surrounding a dental implant with a published clinical observation by Watzak et al. [34] and a numerical prediction by Chou et al. [31]. Figure 9a shows Watzak's clinical observation of density distribution on baboons and figure $9 \mathrm{~b}$ presents Chou et al.'s bone remodelling prediction around a dental implant. The predicted result (with $K=0.0052 \mathrm{~J} / \mathrm{g}$ ) due to the proposed algorithm is re-produced in figure 9c. The overall density distributions are quite similar for three cases in terms of the trabecular-like pattern and the blank area below the implant. This is particularly true when comparing figure $9 \mathrm{~b}$ and figure $9 \mathrm{c}$. Lin $e t$ al's [35] computational prediction indicates that the average density of peri-implant cancellous bone increases throughout the remodelling period, which are in agreement with our results. However, due to the simplification of the finite element model, discrepancies in density distribution do exist between the present computational modelling results and the clinical observation. 


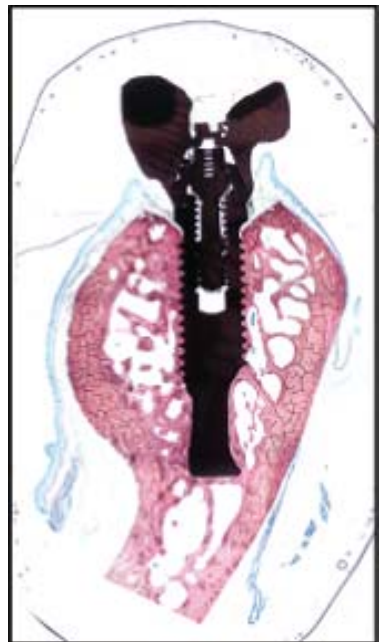

(a)

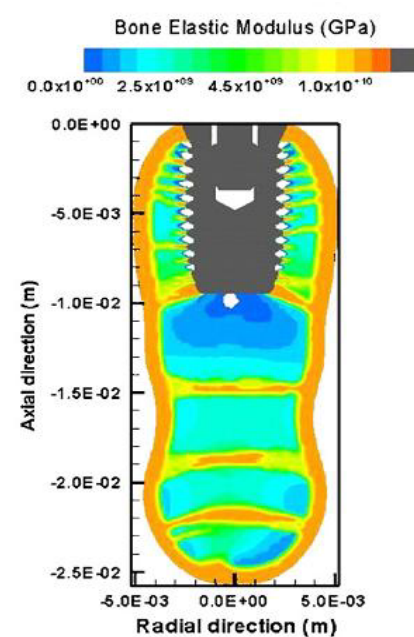

(b)

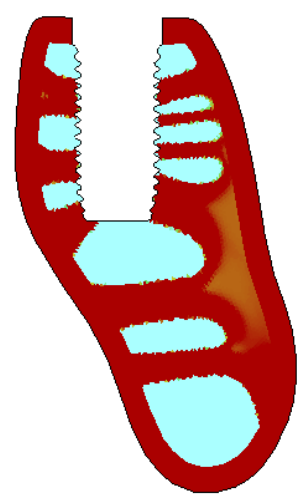

(c)

Figure 9. Comparison of predicted result with clinical observations: (a) density distribution on baboons [34]; (b) Chou et al.'s prediction [31]; (c) predicted result with $K=0.0052 \mathrm{~J} / \mathrm{g}$.

As far as the implant/bone connection is concerned, Roberts [36] found that even 'clinically successful' implants had less than half of the intraosseous interface in direct contact with bone. Block et al. [37] found similar levels of osseointegration for loaded implants in dogs. In this study, a perfect connection between the implant and jawbone is assumed due to the difficulties in accessing accurate clinical data on contact percentage. This simplification on interface modelling may have some influence on the density distribution which should be investigated further.

It should be noted that this study is a preliminary attempt in developing a new bone remodelling algorithm suitable for computer simulation of the highly complex processes of bone healing and remodelling. In addition to the assumed perfect implant/bone connection, this study also employs a simplified and fixed loading condition. This is considerably different from the actual masticatory forces which always vary in both magnitude and direction. Hence, a discrepancy between the resulting density distribution (figure 7) and true anatomy of jawbone is expected. Further work should be done to evaluate the effects of other parameters which may also affect the density distribution in jawbone surrounding a dental implant.

\section{Conclusion}

A new algorithm for the study of bone remodelling in mandibular bone induced by dental implant is proposed in this study, which is a combination and further development of two existing and widely accepted bone remodelling theories. Whilst further optimisation of the proposed algorithm is desirable, our model has been shown to correlate well with clinical observations. An important consideration is that the 'lazy zone' is less important than consideration of the 'strain energy density'. Improved understanding of the factors which influence bone remodelling around osseointegrated dental implants is important in quantifying the forces compatible with maintaining successful osseointegration and preventing overload leading to implant failure. To this end, the newly developed algorithm incorporating elements from previous theories more closely replicates the clinical situation.

\section{Acknowledgement}

The financial support from the 2006 Griffith University Research Grant scheme is gratefully acknowledged. 


\section{References}

[1] Brånemark P I, Hansson B O, Adell R, Breine U, Lindström J, Hallén O, Ohman A 1977 Scand. J. Plast. Reconst. Surg. Suppl. 7 1-132

[2] Schroeder A, van der Zypen E, Stich H, Sutter F 1981 J. Maxillofacial Surgery. 9 15-25

[3] Wolff J L 1982 The law of bone remodelling. Translated by Maquet P, Furlong R in 1986: Springer-Verlag, Berlin

[4] Carter D R 1984 Calcif. Tissue Int. 36 19-24

[5] Huiskes R, Weinans H, Grootenboer H J, Dalstra M, Fudala B, Slooff T J 1987 J. Biomech. 20 $1135-50$

[6] Weinans H, Huiskes R, Grootenboer H J 1992 J. Biomech. 25 1425-41

[7] Turner C H, Anne V, Pidaparti R M V 1997 J. Biomech. 30 555-63

[8] Mullender M G, Huiskes R, Weinans, H 1994 J. Biomech. 27 1389-94

[9] Mullender M G and Huiskes R 1995 J. Orthop. Res. 13 503-12

[10] Brekelmans W A M, Poort H W, Slooff T J J H 1972 Acta Orthop. Scand. 43 301-17

[11] Rybicki E F, Simonen F A, Weis E B 1972 J. Biomech. 5 203-15

[12] Carter D R, Orr T E, Fyhrie D P 1989 J. Biomech. 22 231-44

[13] Huiskes R, Weinans H, van Rietbergen B 1992 Clin. Orthop. Relat. Res. 274 124-34

[14] Kerner J, Huiskes R, van Lenthe G H, Weinans H, van Rietbergen B, Engh C A, Amis A A 1999 J. Biomech. 32 695-703

[15] van Rietbergen B, Huiskes R, Weinans H, Sumner D R, Turner T M, Galante JO 1993 J. Biomech. 26 369-82

[16] Weinans H, Huiskes R, van Rietbergen B, Sumner D R, Turner T M, Galante J O 1993 J. Orthop. Res. 11 500-13

[17] Duyck J, Ronold H J, Van Oosterwyck H, Naert I, Sloten J V, Ellingsen J E 2001 Clin. Oral Implants Res. 12 207-18

[18] Mellal A, Wiskott H W A, Scherrer S S, Belser U C 2004 Clin. Oral Implants Res. 15 239-48

[19] Li J, Li H, Shi L, Fok A S L, Ucer C, Devlin H, Houner H, Silikas N 2007 Dent. Mater. 23 1073-8

[20] Carter D R, Fyhrie D P, Whalen R T 1987 J. Biomech. 20 785-94

[21] Hart R T and Davy D T 1989 Theories of bone modelling and remodelling. 1st ed. Cowin, S.C. Bone Mechanics, CRC Press, Boca Raton

[22] Fyhrie D P and Carter D R 1990 J. Biomech. 23 1-10

[23] Jacobs C R, Simo J C, Bearpre G S, Carter D R 1997 J. Biomech. 30 603-13

[24] Ruimerman R and Huiskes R 2005 Theor. Issues in Ergon. Sci. 6 225-38

[25] Rubin C T and Lanyon L E 1985 Calcif. Tissue Int. 37 411-7

[26] Carter D R and Hayes W C 1977 J. Bone Joint Surg. [Am] 59 954-62

[27] Currey J D 1988 J. Biomech. 21 131-9

[28] Morgan E F, Bayraktar H H, Keaveny T M 2003 J. Biomech. 36 897-904

[29] Rice J C, Cowin S C, Bowman J A 1988 J. Biomech. 21 155-68

[30] Guan H, van Staden R, Loo Y C, Johnson N W, Ivanovski S, Meredith N 2009 Int. J. Oral Maxillofac. Implants 24 866-76

[31] Chou H Y, Jagodnik J J, Muftu S 2008 J. Biomech. 41 1365-73

[32] van Staden R C, Guan H, Loo Y C 2006 Comput. Methods Biomech. Biomed. Engin. 9 257-70

[33] Papavasiliou G, Kamposiora P, Bayne S C, Felton D A 1997 J. Dent. 25 485-91

[34] Watzak G, Zechner W, Ulm C, Tangl S, Tepper G, Watzek G 2005 Clin. Oral Implants Res. 16 408-16

[35] Lin D, Li Q, Li W, Duckmanton N, Swain M 2010 J. Biomech. 43 287-93

[36] Roberts W E 1988 J. Dent. Educ. 52 804-9

[37] Block M S, Finger I M, Fontenot M G, Kent J N 1989 Int. J. Oral Maxillofac. Implants 4 219-25 